\title{
Comparative study of the use of computer assisted navigation system for axial correction in medial unicompartmental knee arthroplasty
}

\author{
Jean-Yves Jenny
}

Received: 5 August 2009/Accepted: 18 May 2010/Published online: 15 June 2010

(C) Springer-Verlag 2010

Dear Editors,

I write you regarding the paper by Lim et al. [2] "Comparative study of the use of computer assisted navigation system for axial correction in medial unicompartmental knee arthroplasty".

Navigation system has been developed to improve the accuracy of implantation of total knee replacement (TKR) [3]. Few systems have implemented an application to unicompartmental knee replacement (UKR) [1]. It is, therefore, important to get an objective evaluation of the systems.

The present study compared two groups of navigated (30 cases) or non-navigated (21 cases) UKR implantation with a well-known navigation system, already validated for TKR. The authors analyzed the accuracy of implantation on post-operative X-rays, and they observed no significant difference between the groups. However, they did not provide any power analysis in their paper. This might be a relevant information, as the number of cases in each group is relatively small. Underpowered study has the risk not to detect a difference which actually exists.

Furthermore, I have a concern about the radiological analysis. The authors used two different techniques for the measurement of the alignment of the lower limb (femorotibial angle and Kennedy protocol). There was a huge discrepancy between the results of the former (10-19\% of the cases within the desired range) and the latter technique (90-95\% of the cases within the desired range). I wonder

J.-Y. Jenny $(\bowtie)$

Centre de Chirurgie Orthopédique et de la Main,

Hôpitaux Universitaires de Strasbourg, 10 avenue Baumann,

67400 Illkirch, France

e-mail: jean-yves.jenny@chru-strasbourg.fr whether both techniques are really adequate if they provide such different results by the same patients.

Finally, we published a couple of years ago a similar study using the same navigation system and a similar radiological evaluation (Jenny et al. [1]). We observed no significant differences in the mean numerical values of all measured angles except for the sagittal orientation of the tibial component, with a significant excessive posterior tibial slope after manual implantation. However, we observed a significant increase in the rate of prostheses implanted in the desired angular range for all criteria except the coronal mechanical femorotibial angle after navigated implantation. We concluded that navigated implantation of a UKR with the used, non-image-based system improved the accuracy of the radiological implantation without any significant inconvenience and with little change in the conventional operative technique. How can the authors explain that they observed so different results?

\section{References}

1. Jenny JY, Boeri C (2003) Unicompartmental knee prosthesis implantation with a non-image-based navigation system: rationale, technique, case-control comparative study with a conventional instrumented implantation. Knee Surg Sports Traumatol Arthrosc 11:40-45

2. Lim MH, Tallay A, Bartlett J (2009) Comparative study of the use of computer assisted navigation system for axial correction in medial unicompartmental knee arthroplasty. Knee Surg Sports Traumatol Arthrosc 17:341-346

3. Picard F, Deakin AH, Clarke JV, Dillon JM, Gregori A (2007) Using navigation intraoperative measurements narrows range of outcomes in TKA. Clin Orthop 463:50-57 